\title{
Pulmonary Embolism an Uncommon First Presenting Symptom of an Undiagnosed Case of HIV
}

\author{
Anil Jha ${ }^{1}$, Hassan Ghoz ${ }^{1}$, Nicholas James ${ }^{2}$ \\ ${ }^{1}$ Division of Internal Medicine, Steward Carney Hospital, Tufts School of Medicine, Dorchester, MA, USA \\ ${ }^{2}$ Division of Critical Care and Pulmonology, Steward Carney Hospital, Tufts School of Medicine, Dorchester, MA, \\ USA
}

\section{ABSTRACT}

A 32 years old male who presented to our emergency department with shortness of breath, cough and weight loss, all started less than a month time and later diagnosed with HIV infection with very high viral load and low CD4 count. His presenting history was lead the diagnostic workup toward malignancy as there was large hilar masses and concern of metastasis to different place mainly abdomen. Suspicion of HIV arouses as the biopsy from the hilar mass came back negative for malignancy but was positive for tuberculosis. HIV serology came back positive. The literature review showed pulmonary embolism as the very rare first presentation of HIV, and only four cases reported so far. Although venous thromboembolism is very common in chronic cases of HIV. J Microbiol Infect Dis 2018; 8(1):33-36

Keywords: HIV, pulmonary embolism, cough, chest pain, tuberculosis

\section{INTRODUCTION}

In 2014, an estimated 44,000 people were diagnosed with the human immunodeficiency virus (HIV) according to the Centers for Disease Control and Prevention (CDC). As of June 2016, more than 1.2 million people are living with HIV in the United States, and 1 out of 8 is not aware of their diagnosis (https://www.cdc.gov/hiv/statistics/index.html).

The most common clinical findings of acute HIV infection are fever, lymphadenopathy, sore throat, rash, myalgia/arthralgia, diarrhea, weight loss and headache $[1,2]$.There are very rare cases of HIV presenting as pulmonary embolism (PE) [3-5]. There is proven increased risk of vascular diseases involving both the arterial and venous systems among HIV-infected patients [67]. HIV is a known prothrombotic condition which increases the risk of venous thromboembolism, with reports showing rates of $0.19 \%-7.63 \%$ per year [7]. The risk factors for PE in HIV-infected patients includes an increase in pro-coagulant factors originating from CD4+ lymphocyte, apoptosis [8] and increased levels of microparticles which initiate the coagulation cascade [9]. Furthermore, reduced levels of antithrombin III, activated protein $\mathrm{S}$ and activated protein $C$ [9-12], acquired heparin cofactor II deficiency [13] and increased levels of fibrinogen, d-dimer, von-Willibrand factor, plasminogen activator inhibitor-1, and tissuetype plasminogen activator antigen [14]. The low CD4 count is another prothrombotic status [15]. Opportunistic infection like Pneumocystis jirovecii in HIV increases the risk of thrombosis due to abnormalities in the hemostatic pathway like antiphospholipid and lupus anticoagulant antibodies [16]. Also, CMV infection has a high affinity for thrombosis by infecting the endothelial cells [17].

These causes have been studied in established, chronic HIV cases. We are presenting here a rare case of newly diagnosed PE and tuberculosis as the presenting diagnosis of undiagnosed HIV case which initially was thought to represent pulmonary malignancy.

\section{CASE REPORT}

A 30-year-old African-American male without past medical history and not on home medication presented to our Emergency department with chief complaints of cough for three weeks and pleuritic chest pain for 1-2 weeks with recent worsening. $\mathrm{He}$ also mentioned feeling fatigued most of the time for 4-6 weeks before presentation with unintentional twenty-pound weight loss. Before the presentation, the patient had seen his PCP a 
few weeks prior and declined blood work as well as high-risk behavior for sexually transmitted infections. On further questioning, he said he lived with his roommate, worked as an event manager, smoked hookah occasionally for last 2-3 years, drinks alcohol socially denied recreational drug use and was sexually active with women infrequently using condoms. His vital signs were significant for tachycardia in 110 s, otherwise afebrile with normal blood pressure and oxygen saturation. His blood work on admission revealed an erythrocyte sedimentation rate of 90 and D-dimer 6.64. Chest X-Ray showed left-sided mass seen in relation to the aorticopulmonary window (Figure 1).

With his presenting complaint of pleuritic chest pain and cough and elevated D-Dimer, pulmonary embolism was evaluated with CT pulmonary angiogram .which showed bilateral pulmonary emboli within bilateral lower lobes, right greater than left (Figure 2a). Also reported was a $5 \times 3 \times 3 \mathrm{~cm}$ mass centered within the aortopulmonary window, essentially contiguous with a $4.5 \times 3.5 \times 3 \mathrm{~cm}$ subcarinal mass and accompanied by additional mild superior mediastinal, left hilar, and bilateral supraclavicular lymphadenopathy (Figure 2b). Furthermore, abdominal images showed a $2.5 \times 1.5 \times 1.8 \mathrm{~cm}$ mass within the upper abdomen, centered at the celiac artery bifurcation and multiple small splenic hypodensities (Figure 2c). These findings raised the concern of lymphoma or primary lung malignancy with metastatic spread. Based on the initial workup, a preliminary diagnosis of lung cancer causing a Pro-coagulant state resulting in pulmonary embolism was made. Lower extremity Doppler ultrasonography was negative. The patient was started on a heparin drip in the emergency department and admitted to the medical floor for further management. An echocardiogram was done which revealed normal ejection fraction, no significant valvular pathology, and normal chambers size. A scrotal ultrasound was done to rule out testicular malignancy which was negative.

A CT-guided percutaneous biopsy was recommended for tissue diagnosis. Given that the mass was central, thoracic surgery was consulted for tissue biopsy. Ultimately, flexible bronchoscopy and cervical mediastinoscopy were performed with biopsy. The frozen section revealed no malignant cells. Final pathology result reported granulomatous lymphadenitis with necrosis. The acid-fast stain was positive for abundant mycobacterial organisms. The fungal stain was negative. The patient was put in airborne isolation, and three sputum AFB smears were obtained which was reported as Mycobacterium species. Four drugs antituberculous treatment was initiated. At this time concern for HIV was raised and HIV tests were sent. To our surprise, HIV 1 antibody came back positive with HIV viral load of 2,060,000 and a CD4 count of 55. The patient was asked again about his sexual health, and he answered that he was sexually active with multiple female partners in last 1-2 years and never used a condom. HIV P24 antigen was non-reactive. Hepatitis $B$ and $C$ testing were also negative. The patient was transitioned to Lovenox and finally to Apixaban for the pulmonary embolism. As mentioned earlier, antitubercular therapy with four medications was started before the HIV diagnosis. Later patient was transferred to an Infectious Disease specialized hospital for initiation of HIV treatment.

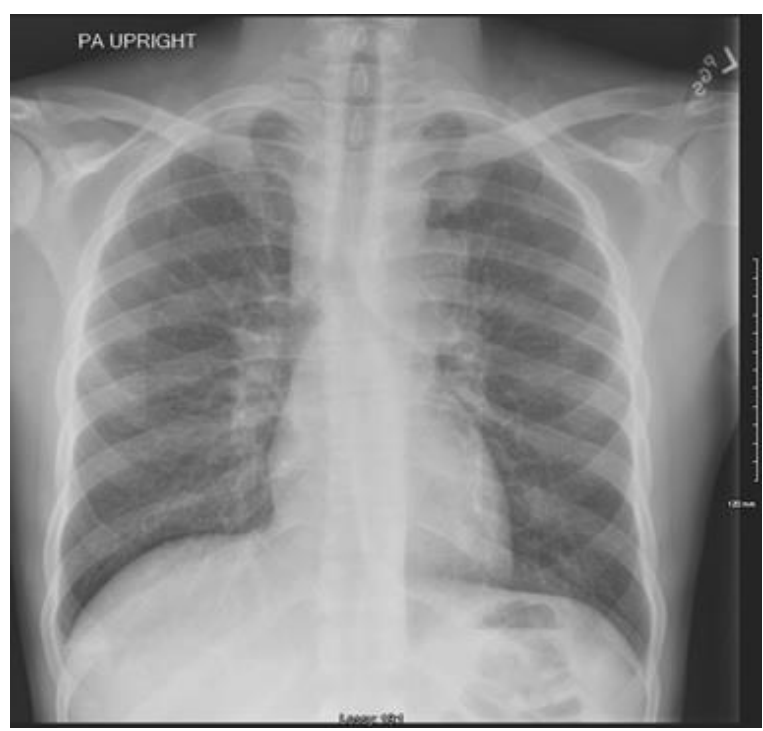

Figure 1- Chest X-Ray showing left-sided mass seen in relation to the aorticopulmonary window.

ESR was $90 \mathrm{~mm} / \mathrm{h}$, HIV1 antibody positive, viral load 2,060,000 cells $/ \mathrm{mm}^{3}$, absolute CD4 count 55.

He was started on four drug regimen for tuberculosis. He was also started on Bactrim 800-160 mg p.o. daily for prophylaxis. For pulmonary embolism, he was started on Heparin drip and later transitioned to Lovenox and finally 


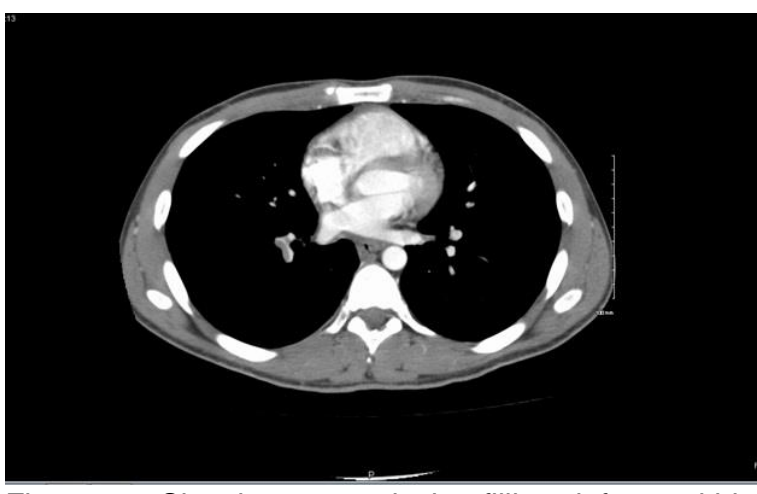

Figure 2a. Showing nonocclusive filling defects within several segmental and subsegmental pulmonary arterial vessels supplying bilateral lower lobes, right greater than left.

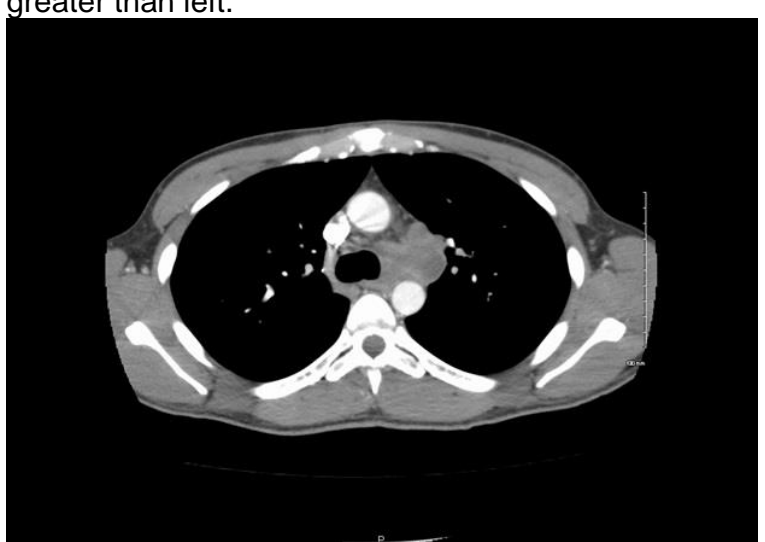

Figure $2 \mathrm{~b}$. There is a $5 \times 3 \times 3 \mathrm{~cm}$, lobulated, heterogeneous, soft tissue density mass centered within the aortopulmonary window, extending laterally into the medial portion of the left upper lobe and abutting but not invading the large vessels or the large airways. Essentially contiguous with this mass is a $4.5 \times 3.5 \times 3 \mathrm{~cm}$ similar-appearing subcarinal mass.

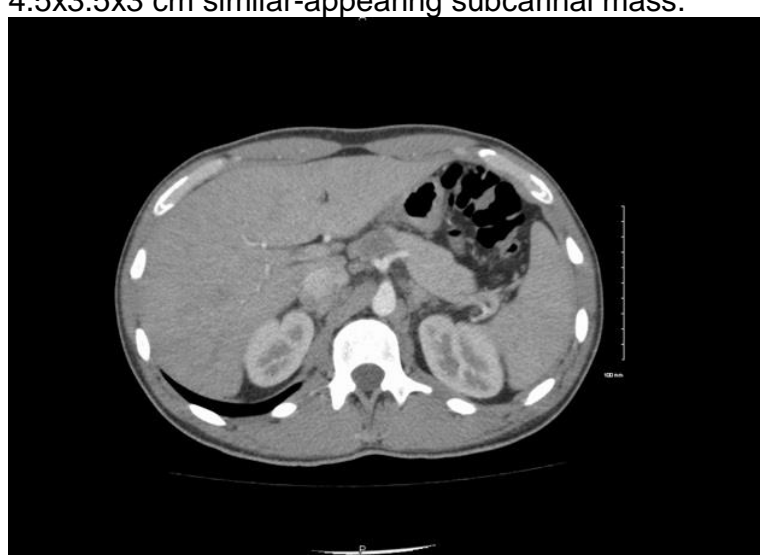

Figure 2c. The included upper abdomen demonstrates multiple small hypodense splenic lesions and a $2.5 \times 1.8 \times 1.8 \mathrm{~cm}$ hypodense mass centered at the bifurcation of the celiac artery and indenting the superior portion of the proximal pancreatic body.

to Apixaban $10 \mathrm{mg}$ p.o. twice daily for the pulmonary embolism. He was transferred to infectious disease hospital for initiation of antiretroviral therapy.

At the Infectious disease hospital, HIV medications were initiated and the patient was later discharged on both antitubercular and antiretroviral treatment.

In this case, the patient was not expecting this diagnosis as he considered himself low risk for contracting the disease. It was devastating news for him and his dear one. His girlfriend was also concerned as she was in a physical relationship with him and never used barrier contraception.

\section{DISCUSSION}

There are several case reports and studies about venous thromboembolism and pulmonary embolism risk in HIV infected patients [6-7]. These studies have shown that HIV infection is a hypercoagulable state, making patients prone to thromboembolic events. The cause of thrombosis in HIV infection is multifactorial, including opportunistic infection, HIV-related malignancies [18,19] and intravenous drug use [6], increased the level of procoagulant factors, decreased the level of protein $\mathrm{C}$ and $\mathrm{S}$, factor $\mathrm{V}$ Leiden mutation, lupus antibody presence. An association between tuberculosis and HIV infection is also well known [20-22]. We have presented a case of pulmonary embolism, which was the sole presentation of underlying acute HIV infection and tuberculosis initially believed to represent malignancy. On admission, the patient's coagulation profile was normal, although we were unable to send markers of hypercoagulability as the patient was started on a heparin drip in the emergency department. CT-PA being the gold standard in diagnosing pulmonary embolism, we had a positive result for PE in our case but it raised the concern of primary lung malignancy or lymphoma, both well-established causes of thromboembolism. The CT Pulmonary Angiogram finding delayed the diagnosis of HIV in our case. There were no typical symptoms of HIV except significant weight loss.

High suspicion for HIV infection should always be raised in a young patient with no typical symptoms of HIV presenting with a thromboembolic event.

\section{CONCLUSION}

Anamnesis is always an important and a basic tool to set the path to right diagnosis. HIV should 
be considered as differential even in absence of typical presentation.

HIV is a procoagulant state but mostly the episodes of venous thromboembolism occur after few months of diagnosis and frequently after starting of HAART. Although it may present severe complications like pulmonary embolism.

\section{ACKNOWLEDGMENTS}

Conflict of interest: The authors declare no personal or financial conflict of interest.

Financial Disclosure: No financial support was received.

\section{REFERENCES}

1.Cooper DA, Gold J, Maclean P, et al. Acute AIDS retrovirus infection. Definition of a clinical illness associated with seroconversion. Lancet Lond Engl 1985; 1 (8428):537-540.

2.Tindall B, Barker S, Donovan B, et al. Characterization of the Acute Clinical Illness Associated With Human Immunodeficiency Virus Infection. Arch Intern Med 1988; 148(4):945-949.

3.Patra S, Nagesh C, Reddy B, Srinivas B, Manjunath $\mathrm{C}$, Hegde $\mathrm{M}$. Acute pulmonary embolism being the first presentation of undetected HIV infection: report of two cases. Int J STD AIDS 2013; 24(6):497-499.

4.Nagaraja V, Terriquez JA, Gavini H, Jha L, Klotz SA. Pulmonary Embolism Mimicking Pneumonia in a HIV Patient. Case Rep Med 2010; 2010: 394546/

5.Howling SJ, Shaw PJ, Miller RF. Acute pulmonary embolism in patients with HIV disease. Sex Transm Infect 1999; 75(1):25-29.

6.Rasmussen L, Dybdal M, Gerstoft J, et al. HIV and risk of venous thromboembolism: a Danish nationwide population-based cohort study. HIV Med 2011 1; 12(4):202-210.

7.Klein SK, Slim EJ, de Kruif MD, et al. Is chronic HIV infection associated with venous thrombotic disease? A systematic review. Neth J Med 2005; 63(4):129136.

8.Embretson J, Zupancic M, Ribas JL, et al. Massive covert infection of helper $\mathrm{T}$ lymphocytes and macrophages by HIV during the incubation period of AIDS. Nature 1993; 362(6418):359-362.

9.Gris JC, Toulon P, Brun $S$, et al. The relationship between plasma microparticles, protein $S$ and anticardiolipin antibodies in patients with human immunodeficiency virus infection. Thromb Haemost 1996; 76(1):38-45.

10.Grinnell BW, Joyce D. Recombinant Human Activated Protein C: A System Modulator of Vascular Function for Treatment of Severe Sepsis. Crit Care Med 2001: 29 (7 Suppl), S53-S60.
11.Toulon $P$, Lamine $M$, Ledjev I, et al. Heparin cofactor II deficiency in patients infected with the human immunodeficiency virus. Thromb Haemost 1993; 70:730-735.

12.Erbe M, Rickerts V, Bauersachs RM, Lindhoff-Last E. Acquired protein $\mathrm{C}$ and protein $\mathrm{S}$ deficiency in HIVinfected patients. Clin Appl Thromb Hemost 2003; 9:325-331.

13.Bertina RM, van der Linden IK, Engesser L, Muller HP, Brommer EJ. Hereditary heparin cofactor II deficiency and the risk of development of thrombosis. Thromb Haemost 1987; 57(2):196-200.

14.Schved JF, Gris JC, Arnaud A, et al. von Willebrand factor antigen, tissue-type plasminogen activator antigen, and risk of death in human immunodeficiency virus 1-related clinical disease: independent prognostic relevance of tissue-type plasminogen activator. J Lab Clin Med 1992; 120:411419.

15.Saif MW, Bona R, Greenberg B. AIDS and Thrombosis: Retrospective Study of 131 HIV-Infected Patients. AIDS Patient Care STDs 2001; 15(6):311320.

16.Aboulafia DM, Mitsuyasu RT. Hematologic abnormalities in AIDS. Hematol Oncol Clin North Am 1991; 5(2):195-214.

17.Smith KJ, Skelton HG, Yeager J, Wagner KF. Cutaneous thrombosis in human immunodeficiency virus type 1-positive patients and cytomegalovirus viremia. Arch Dermatol. 1995; 131:357-358.

18.Doberson MJ, Kleinschmidt-DeMasters BK. Superior sagittal sinus thrombosis in a patient with acquired immunodeficiency syndrome. Arch Pathol Lab Med 1994; 118:844-846.

19.Kaufmann T, Nisce LZ, Metroka C. Thromboembolism in AIDS-related Kaposi's sarcoma. JAMA 1991; 266(20):2834.

20.Selwyn PA, Hartel D, Lewis VA, et al. A prospective study of the risk of tuberculosis among intravenous drug users with human immunodeficiency virus infection. N Engl J Med 1989; 320:545-550.

21.Day CL, Mkhwanazi N, Reddy S, et al. Detection of Polyfunctional Mycobacterium tuberculosis-Specific T Cells and Association with Viral Load in HIV-1Infected Persons. J Infect Dis 2008; 1;197:990-999.

22. Raviglione MC, Harries AD, Msiska R, Wilkinson $D$, Nunn P. Tuberculosis and HIV: current status in Africa. AIDS Lond Engl 1997; 11 Suppl B:S115-123. 